\section{Freezing Techniques: History, Comparisons, and Applications}

Bill Graham, Bibst Labs Brookline, $\mathrm{NH}$

Jotham R. Austin II, University of Chicago Chicago, IL

Andres Kaech, University of Zurich, Switzerland

John E. Heuser, Washington Univ. School of Med., St. Louis, MO bill@bibst.com

Specimen preparation techniques have evolved hand in hand with microscopy since the first microscopes. Since the introduction of the first Electron Microscope (EM) in the 1930's, the basic problem with biological electron microscopy has been to preserve the structure of soft condensed, hydrated matter (e.g. tissues, cells, proteins, etc.) so that they can be viewed in the harsh environment of the electron microscope's high vacuum and ionizing radiation. For this, cells must be "fixed" with chemical cross-linkers, commonly glutaraldehyde, formaldehyde or some combination of both, stained with heavy metals (osmium tetroxide that provides contrast of biological components), dehydrated with an organic solvent, and infiltrated with a resin for eventual thin-sectioning (1). Only then can it be viewed with the EM. Such treatment with chemical fixatives and stains remains the standard approaches to arrest biological processes in cells or tissues, but at the cost of introducing clearly recognizable artifacts.

EM research in pathology, descriptive anatomy, ultrastructure, etc. has employed such preparation techniques many decades. However, researchers have found that chemical fixatives act slowly (seconds to minutes) and in many cases do not preserve cellular processes and components without introducing artifacts. Moreover, this slow rate of fixation, combined with room temperature dehydration of the specimen, severely perturbs the physical properties of biological membranes, often leading to profound distortions in the shape, volume, and contents of cells and their components.

Yet, despite its many trade-offs, this sort of approach to EM became recognized as the only method to detect and illustrate cellular structures at high resolution, down to $\mathrm{nm}$ resolutions, within the context of complex biological systems (2). Over the last four decades of the twentieth century, EM images yielded information that led to significant discoveries in medicine, science and biology (i.e.: detecting viral and bacterial infections, disease states, imaging organelles within cells, hydrated materials, etc.).

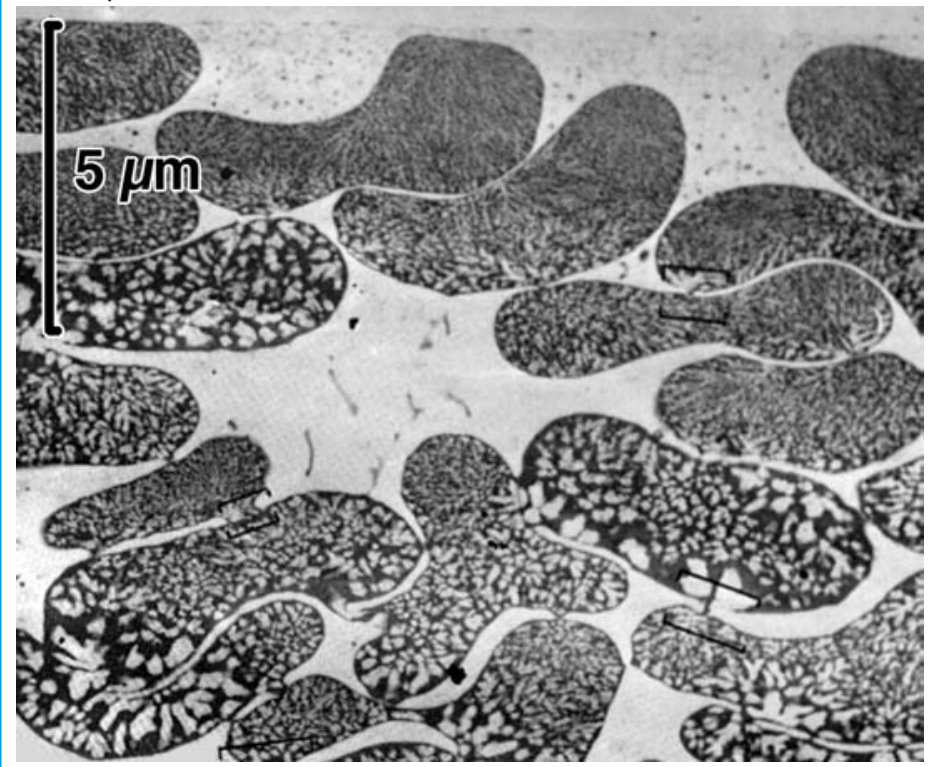

Figure 1: Human red blood cells, conventionally plunge-frozen by hand, illustrate the gradient of ice crystals that is unavoidable by this relatively crude approach. The ice crystals obviously grow increasingly larger with depth. (Courtesy JH)
In the 1970's, a new era of EM began with the introducuvi ur ur first cryo-preservation techniques. "Freeze fracturing" was developed as a means to provide three-dimensional views of biological materials. This technique involved freezing a biological sample, fracturing it open in vacuo, and depositing heavy metals onto its freshly cleaved surfaces. The resultant images revealed splitting and high-resolution replication of lipid bilayers. Initially, the freezing protocols used for immobilizing biological structures in this technique were prone to create the recognizable artifact of ice crystal formation. (Figure 1). As a result, cryoprotectants such as glycerol were utilized to prevent or at least minimize such ice crystal formation. These early freezing methods, some of which are still in use today, consist of plunging samples mounted on thermally conductive supports into containers of liquefied organic gases (chlorodifluoromethanes, ethane, propane), and/or slurries of liquid nitrogen.

Again, since the freezing rates that could be achieved by handplunging into cryogenic liquids were slow and variable, and were unable to prevent ice crystal formations without chemical pretreatment with fixatives and cryoprotectants, they remained prone to serious artifacts, and led many investigators to question the results and interpretations of these new cryo-preservation techniques. This led to a decline in EM, with many concluding that EM had provided all it could about the organization of the cell, and further understanding would require molecular biology, genetics and nanotechnology techniques. EM also began to be compared unfavorably with the advanced new techniques of fluorescent light microscopy that were becoming available in the '90's.

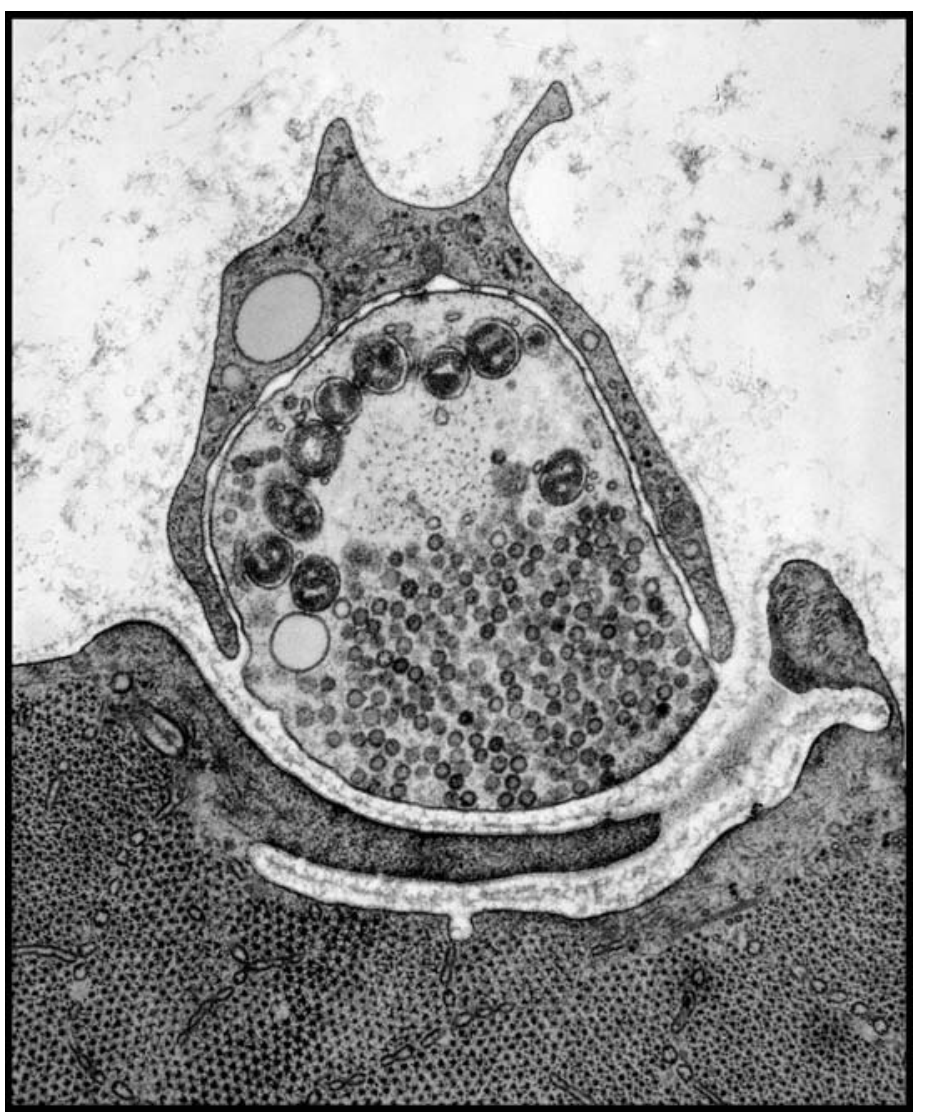

Figure 2: $\quad$ Optimally preserved motor nerve terminals from frog muscles frozen by "slam"-freezing against a copper block cooled to liquid helium temperature. The high quality of freezing is reflected for example in the clear details seen in the dark mitochondria (around its upper circumference), and the excellent preservation of its large pool of extremely delicate 'synaptic vesicles' (the small spheres of varying darkness that fill its lower portions). The motor nerve terminal is 'capped' by a thin cell process of the so-called Schwann Cell, and nestles down within a folded furrow of the muscle cell below (Courtesy JH) 


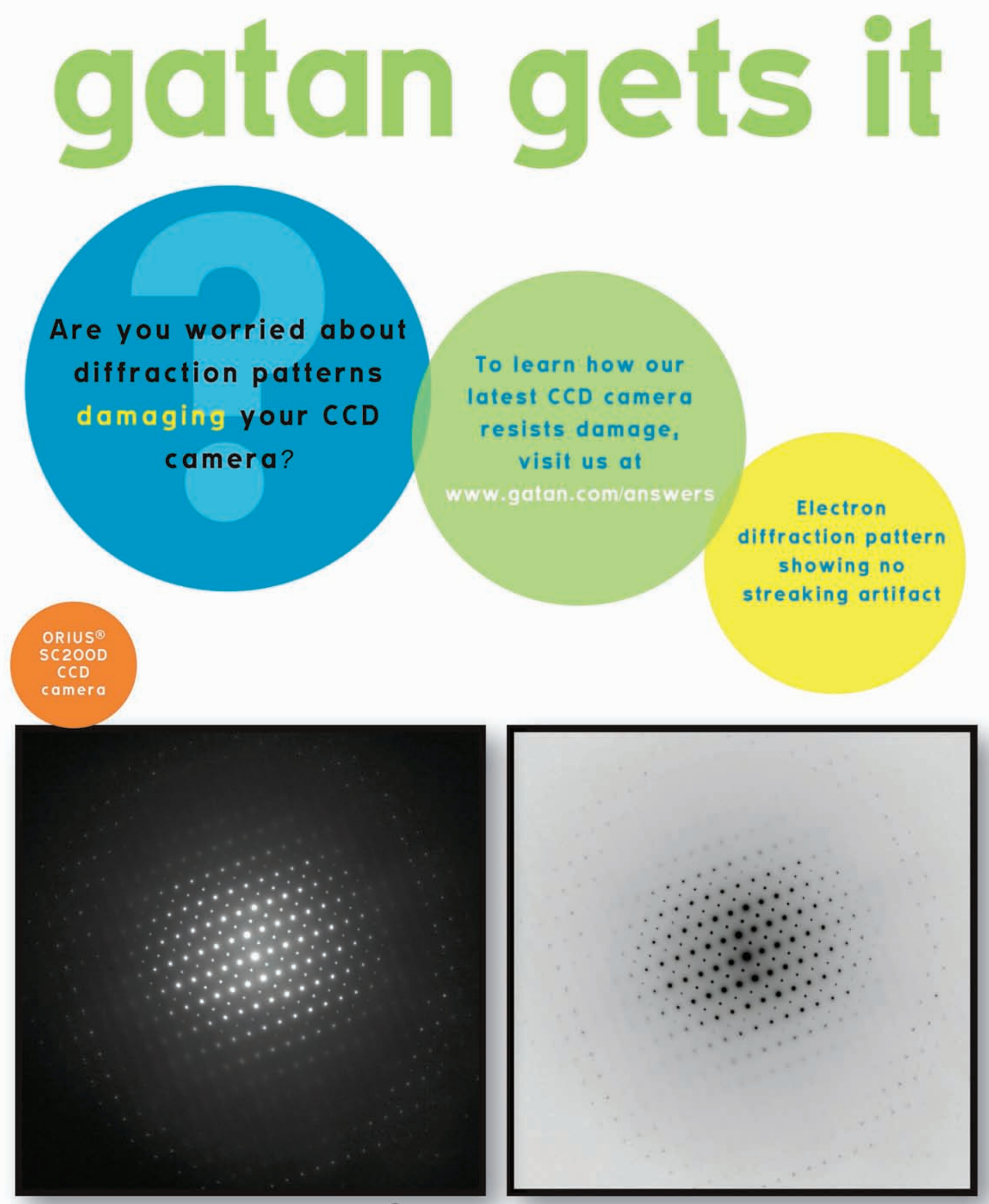

$\mathrm{LaCO}_{3}[110]$ electron diffraction pattern recorded with Gatan ORIUS ${ }^{\oplus}$ SC200D CCD camera at 200kV. (Left) as recorded; (right) contrast reversed. 


\section{Cryofixation}
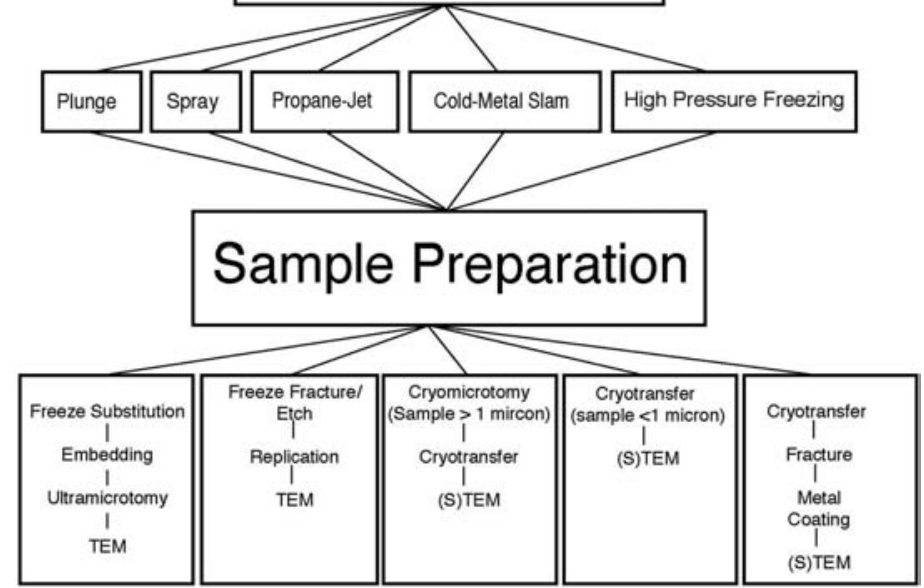

Drawing 1: Flow chart with common options illustrates

Cryofixation pathways to Sample Preparation that routinely yield EM images of interest.

We would argue that EM research of the last 20+ years has indeed continued to expand our understanding about the cell, cell-cell interactions, molecular machinery, genomes, drug interactions, bio- and eco- engineered materials, etc. We would argue that it will never be possible to fully understand the dynamic nature of the cell without being able to see what is going on in it. Without EM, this intrinsic cellular context would be missing.

Fortunately, today we possess the ability to design experiments around relevant questions and combine them with the power of cryoEM. The ability to cryo-fix cells instantaneously, and thereby eliminate all the artifacts of chemical fixation, combined with cutting-edge advances in electron microscopes and techniques (such as 3D electron tomography, immunocytochemistry, and correlative light-EM studies) has opened a new era of EM studies. Meaningful correlation of structure and function is the reward of this new approach. (Figure 2)

Today, several different rapid freezing methodologies for specimen preparation are used, all of which strive to instantaneously halt activity in living cells and tissues and capture them in their native state. The desired goal of cryofixation is to create vitrification of all the water in biological tissues; specifically, to convert tissue water into the amorphous solid state, and thereby not to perturb the interactions of cell constituents at all (3).

Successful freezing methods today include: Spray and plunge freezing (4), Contact / Mirror block freezing (5), Propane Jet freezing (6), and High pressure freezing (7). Each of these methods has certain

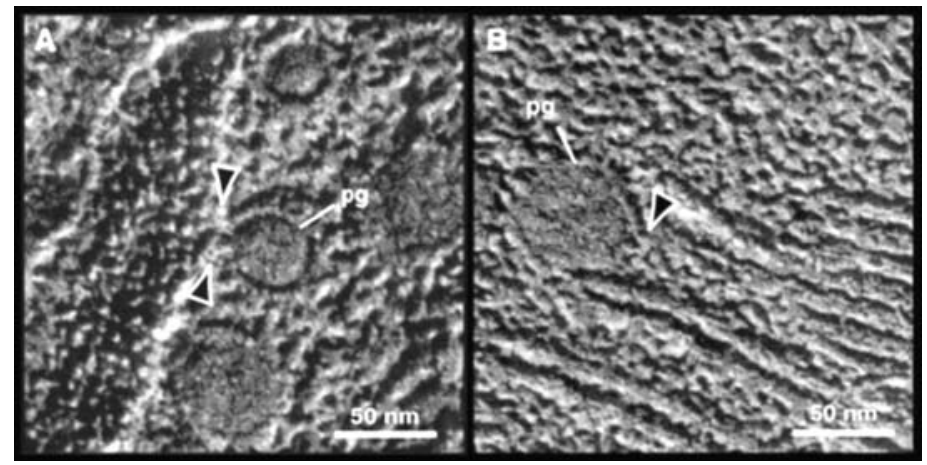

Figure 3: Two examples of freeze fractured chloroplasts that were initially plunge frozen. In both images, the half-lipid bilayer that surrounds the lipid core has been partially exposed due to the freeze-fracturing technique, revealing the neck-like connection between the plastoglobule and thyalkoid membrane. pg: plastoglobule. (Courtesy JA)

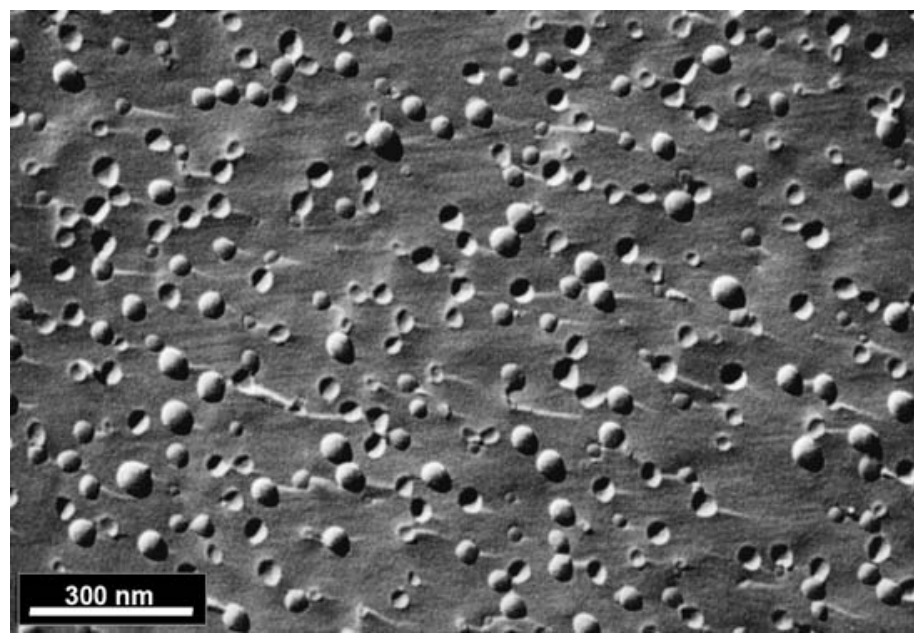

Figure 4: Liposomes: Freeze-fracture replica of a liposome suspension. Propane-jet frozen, freeze-fractured and coated with $2 \mathrm{~nm}$ of $\mathrm{Pt} / \mathrm{C}$ at $45^{\circ}$ and with $20 \mathrm{~nm}$ of carbon by electron beam evaporation. Courtesy (AK) Electron Microscopy Center Zurich (EMEZ), ETH Zurich, Switzerland.

advantages, benefits, and limitations. Cutting-edge researchers, as well as today's equipment manufacturers have produced an array of instrumentation that is widely used and commercially available. When combined with cryo-preservation techniques, a myriad of EM-imaging possibilities emerge. (Drawing 1)

Choosing between freezing methods is often determined by experimental design, and /or the availability of operational equipment. There are many different types of commercial units in the field, products of creative suppliers and ingenious laboratories. Given the detailed and complex issues associated with various techniques, the novice is encouraged to seek out and train with experienced practitioners. Perhaps 200+ laboratories worldwide are engaged in routine cryo-work. These labs are producing a new generation of researchers, thus creating many exciting opportunities for new laboratories and new commercial applications.

Plunge Freezing is the controlled immersion of small, thin samples into liquefied organic gases, typically liquid propane. Cooling rates at the specimen surface are reportedly 10003000 degrees $\mathrm{C}$ per second. Very small samples are typically mounted on $3 \mathrm{~mm}$ EM grids, held by forceps in a free-falling device and plunged by gravity into liquid regions of the cryogen. This method is compatible with various post-preparation techniques, including freeze-fracture and cryoTEM. By this approach, properly frozen specimens display good freezing to a depth of typically $\sim 1-3 \mathrm{mi}-$ crons (Figure 3).
Drawing 2: Schema shows the 'business end' or sample-holder of the Heuser "slam-freezer", whereupon is placed a muscle with its nerve still intact. This is wrapped around wires that will provide a stimulus precisely as the muscle is rapidly frozen. The liver slice adds cushioning to minimize deformation of the muscle by mechanical compression during impact or "slamming".

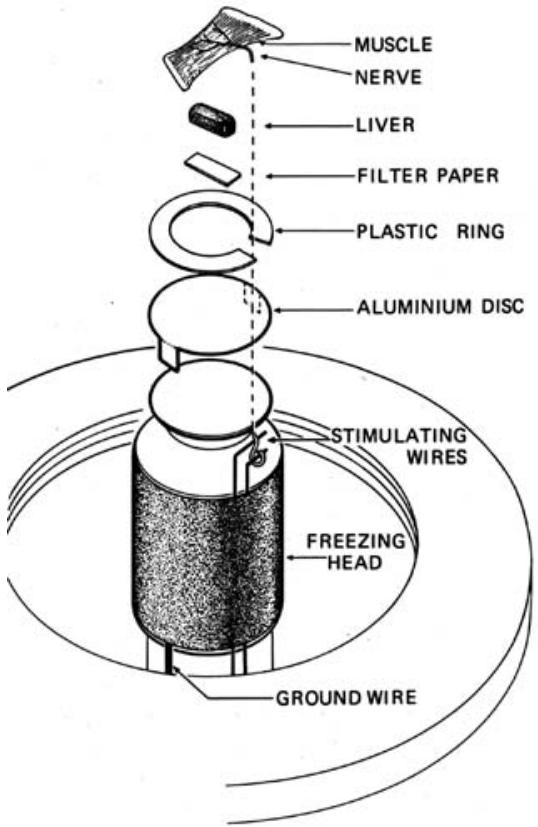


Mini-SEM Compact Footprint ( $22 \times 22 \times 22$ inches)

$15 \mathrm{keV}$ or $30 \mathrm{keV}$ Analytical Miniature Electron Microscope

120,000 X - Magnification (Digital Zoom)

Liquid Nitrogen Free - Light Element X-ray Detector

Easy Spectral Identification

Advanced Image Analysis \& Image Processing

Ultra Fast Elemental Mapping - Spectra for every pixel

Auto Report Writing

Installation \& Training in Minutes!

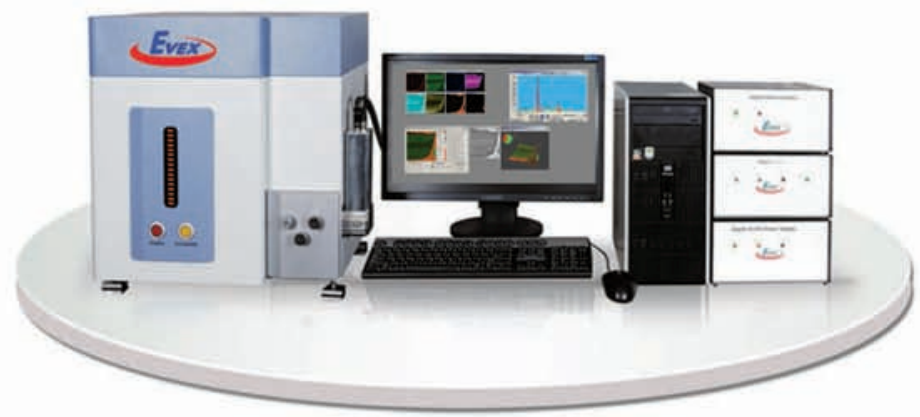

Scanning Electron Microscope

X-ray NanoAnalysis

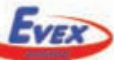

\section{Call Today}

\section{$609-252-9192$}

\section{Research Scientist \\ Laboratory for MultiScale Imaging \\ Stevens Institute of Technology \\ Hoboken, New Jersey USA}

The Stevens Institute of Technology seeks to fill a newly created position of Research Scientist for its Laboratory for Multiscale Imaging (LMSI). The LMSI is a 3000 sq. ft. laboratory housing an analytical FEG TEM/STEM, a FEG SEM, a confocal microscope, and an AFM, among other instrumentation. More information can be found at: www.stevens.edu/hydrogel/libera/research/LMSI/LMSI. html. The LMSI serves a growing community of research students and staff from Materials Science, Chemical Engineering, Mechanical Engineering, Environmental Engineering, Biomedical Engineering, Chemistry, and Chemical Biology, among others, as well as a range of academic and industrial users from off-campus. Current research themes include soft materials, cell-material interactions, biofilms, self assembly, patterning, and nanostructured particulate/surfaces. In addition to pursuing collaborative research, the LMSI Research Scientist will oversee all aspects of running the LMSI including training, maintenance, research execution, and educational outreach. $\mathrm{He} / \mathrm{she}$ will participate in the development of new LMSI initiatives in concert with an interdisciplinary LMSI Advisory Board and the LMSI Director, Prof. Matthew Libera. The initial appointment will be for two years. Qualified candidates will hold a PhD or have equivalent experience in either a physical science/engineering or a life-science discipline. Interested candidates should submit by email, with the subject heading of LMSI RESEARCH SCIENTIST, a detailed resume that includes a clear description of the candidate's experience with various microscopies as well as the contact details of at least three references to Ms. Nancy Webb at Nancy.Webb@stevens.edu.
BEST IN CLASS SERVICE AT REASONABLE PRICES HPM010 HIGH PRESSURE FREEZING SALES AND SERVICE AUTHORIZED MANUFACTURING REP FOR RMC PRODUCTS NEW TO MARKET HIGH VACUUM SPUTTER COATER (USA)

BALZERS/BAL-TEC BAF400 \& 301 FREEZE FRACTURE UNITS

BALZERS/BAL-TEC EM PREP EQUIPMENT SERVICE

ULTRAMICROTOME SALES AND SERVICE

STANDARD AND CUSTOMIZED SERVICE CONTRACTS

FREEZE FRACTURE REPLICA MAKING SERVICE

FIELD SERVICE, REPAIRS, TRAINING, CUSTOMER SUPPORT CONSUMABLES, SUPPLIES, TARGETS, GLASS CHAMBERS

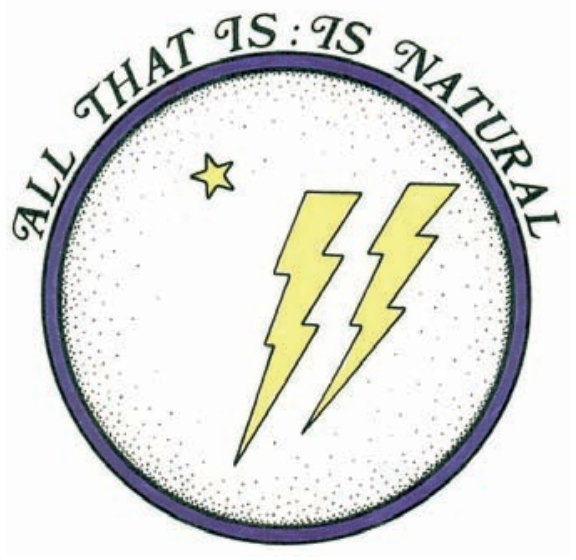

BIBST LABS 603-672-4060

bill@bibst.com

WWW.BIBST.COM 


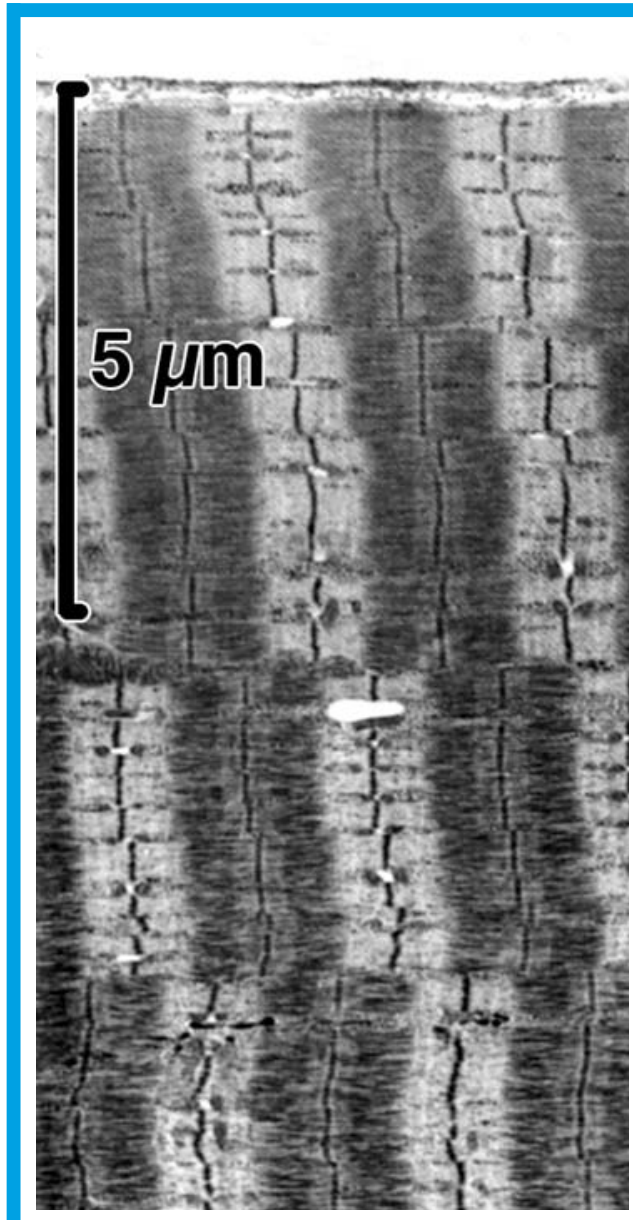

Figure 5: $\quad$ Frog muscle illustrating the typical gradient of freezing achieved by "slam-freezing" against a cryogenic metal block. Ice crystals grow with depth into the tissue. The very best quality of highest density material is found only to 1-2 micron of depth. Distortion and compression of muscle thick and thin filaments, and overall disruption of native tissue architecture, is observed as one moves progressively inward (downward in this view). (Courtesy JH)
Propane Jet Freezing uses opposed metal specimen supports (typically copper) that sandwich a thin aqueous sample, which is then rapidly frozen by blowing liquid propane at the supports from opposite directions. Freezing is performed at normal atmospheric pressure (usually in a fume hood), and require an automated instrument with services including liquid nitrogen, gaseous nitrogen, and propane. Cooling rates at the specimen surface exceed plunge freezing, and are estimated to be 30,000 degrees $C$ per second. Samples $\sim 10$ microns thick can be handled by this approach, so it is useful for achieving reasonably good freezing of suspensions of cells, bacteria, viruses, liposomes, and other liquids. (Figure 4) Additionally, this method adapts well to the "double replica technique" of freeze-fracture.

Rapid Contact Freezing of hydrated materials with cold metal surfaces polished to a mirror finish is also conducted at atmosphere, and also accommodates a wide range of plant and animal samples. The best regions are at the specimen surface, where cooling rates can reach as high as $\sim 100,000$ degrees C per second. Liquid nitrogen or liquid helium cools the metal mirror. Besides the optimally frozen surface regions, reasonably high quality freezing can be achieved by this approach to depths of 5-10 microns. This method can accommodate $1 \mathrm{~cm}$ diameter-size samples. (Drawing 2) Micrographs reveal evidence of the so-called 'freezing front'. (Figure 5) This passes through the sample as heat is withdrawn from it, yielding a progressive deterioration in ultrastructure and increasingly larger ice crystals in deeper regions. This method, also referred to as "slamming", is unique in particular because it is particularly amenable to dynamic studies of cell function. Since the freezing is so abrupt and so precisely timed, it can be synchronized to electrical stimulation of nerve and muscle, for example, or to drug-delivery or other sorts of stimulus-induced events, thereby permitting capture of discrete events on millisecond time scale. Adding "deep-etching" and "rotary replication" to the basic technique of freeze fracture that is commonly used to prepare such "slam"-frozen samples yields remarkable threedimensional views of tissue fine structure. It is also possible to view such "slam"-frozen samples after freeze substitution, or by cryo-SEM, or by a variety of other post-freezing specimen preparation techniques.

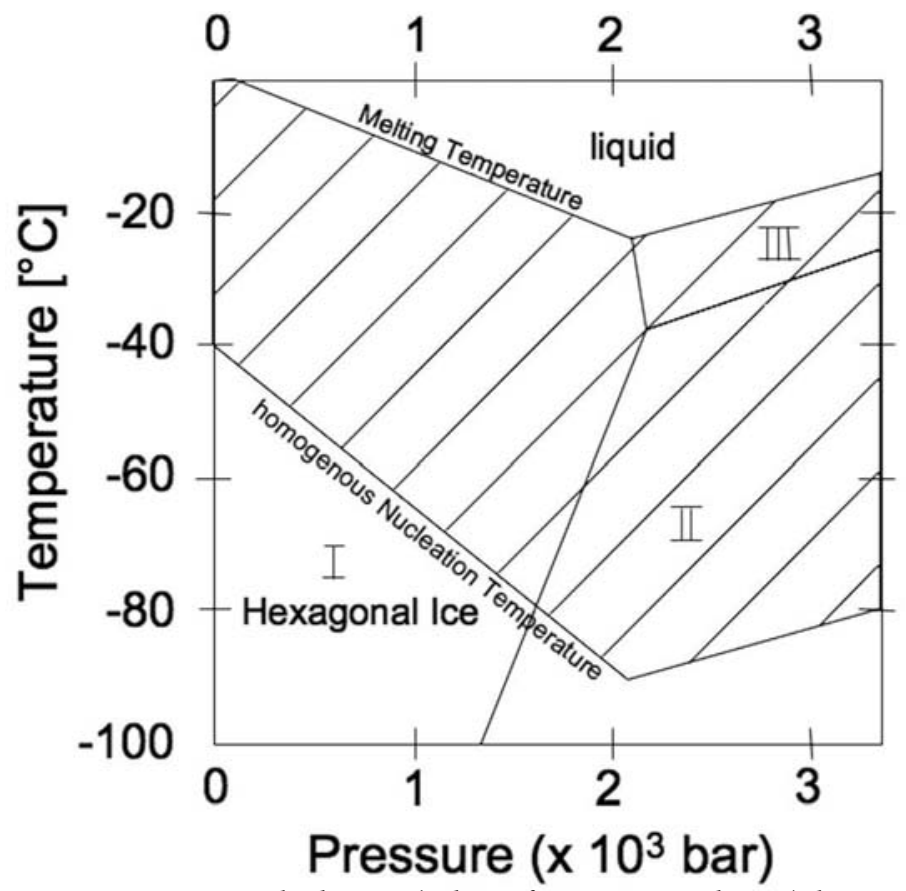

Drawing 3: The diagram (redrawn from Kanno, et.al. 1975) shows the stable state of water as a function of pressure and temperature. (8) Melting temperature and homogeneous nucleation temperature reach a minimum at 2100 bar. Water can be supercooled to $-90 \mathrm{C}$ at 2100 bar, turning it into a very viscous liquid. I, II, \& III are forms of hexagonal ice.

High Pressure (HP) Freezing is Liquid Nitrogen jet freezing of samples held in $3 \mathrm{~mm}$ diameter metal sandwiches, carried out at 2100 bar pressure. Under HP conditions, the freezing and melting points of water, respectively, are lowered, permitting supercooling of the water

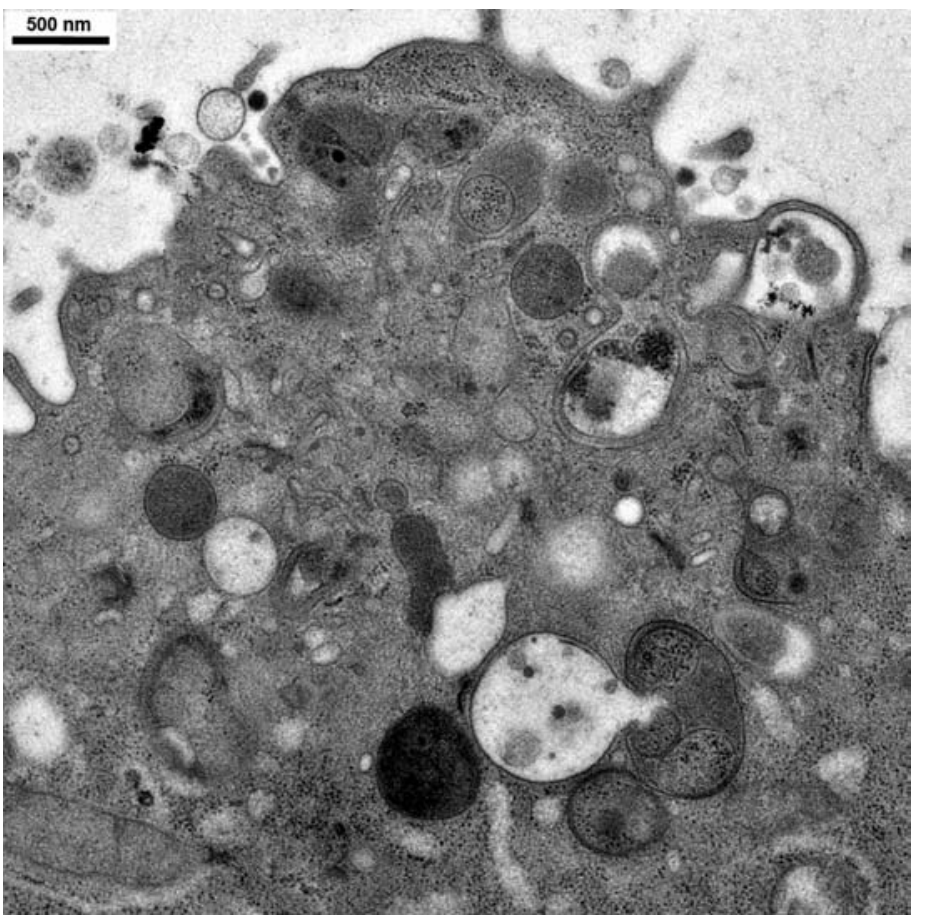

Figure 6: $\quad$ Macrophage: Thin section of macrophage, monolayer on Sapphire disc. High-pressure frozen in its native state (no chemical pretreatment) with a Bal-tec HPM 010 high-pressure freezer, freezesubstituted in acetone with $2 \% \mathrm{OsO}_{4}$, embedded in Epon/Araldite, thin sectioned and stained with uranyl acetate $2 \%$ and Reynolds lead citrate. Courtesy (AK) Electron Microscopy Center Zurich (EMEZ), ETH Zurich, Switzerland. 


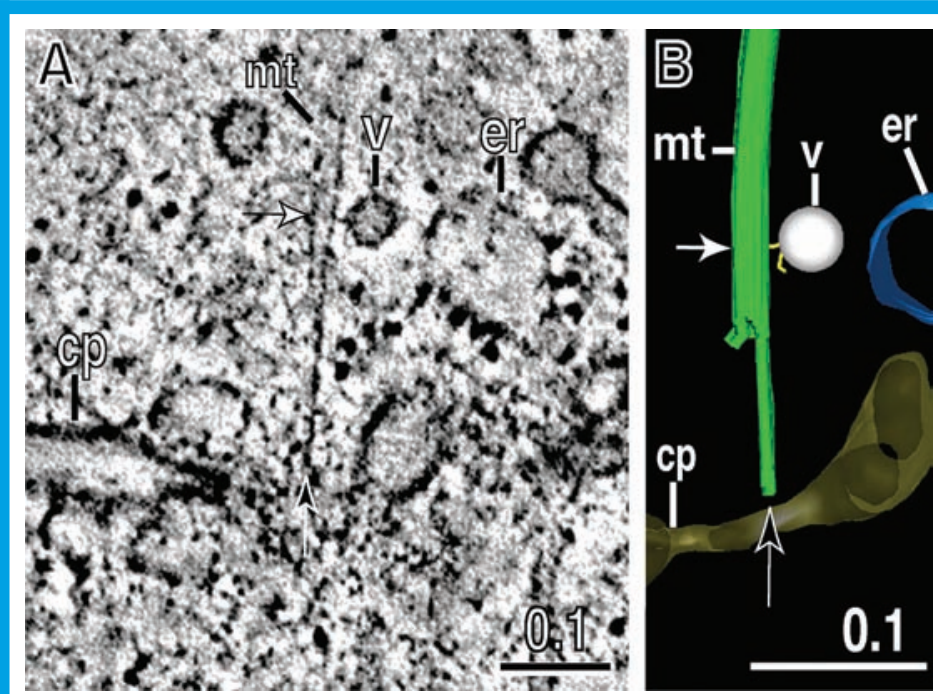

Figure 7: By avoiding the ultrastructural artifacts of chemical fixation by using High Pressure Freezing, it is possible to take full advantage of 3 D electron tomography's 5-7nm resolution. (A) 3 D electron tomographic image of a High Pressure Frozen/Freeze Substituted plant root meristem cell undergoing cytokinesis. During cytokinesis vesicles (v) travel along microtubules $(\mathrm{mt})$ with the aid of a kinesin like motor protein (white arrows). Also shown is a protofilament indicative of a growing microtubule. cell plate (cp); endoplasmic reticulum (er). (B) Tomographic model of Figure A. (Courtesy JA)

inside tissues and eventual homogeneous nucleation of freezing at -90 degree C. At these pressures and temperatures, the viscosity of water is increased by $\sim 1500$ times, which also greatly reduces nucleation and growth of ice crystals. As a result, extremely high rates of cooling are not required, compared to all the approaches that are carried out at one atmosphere. Hence, the comparatively sluggish cooling rate of 200 degrees $\mathrm{C}$ per second is quite adequate. (Drawing 3) By this approach, a wide range of biological samples can be well-frozen, generally

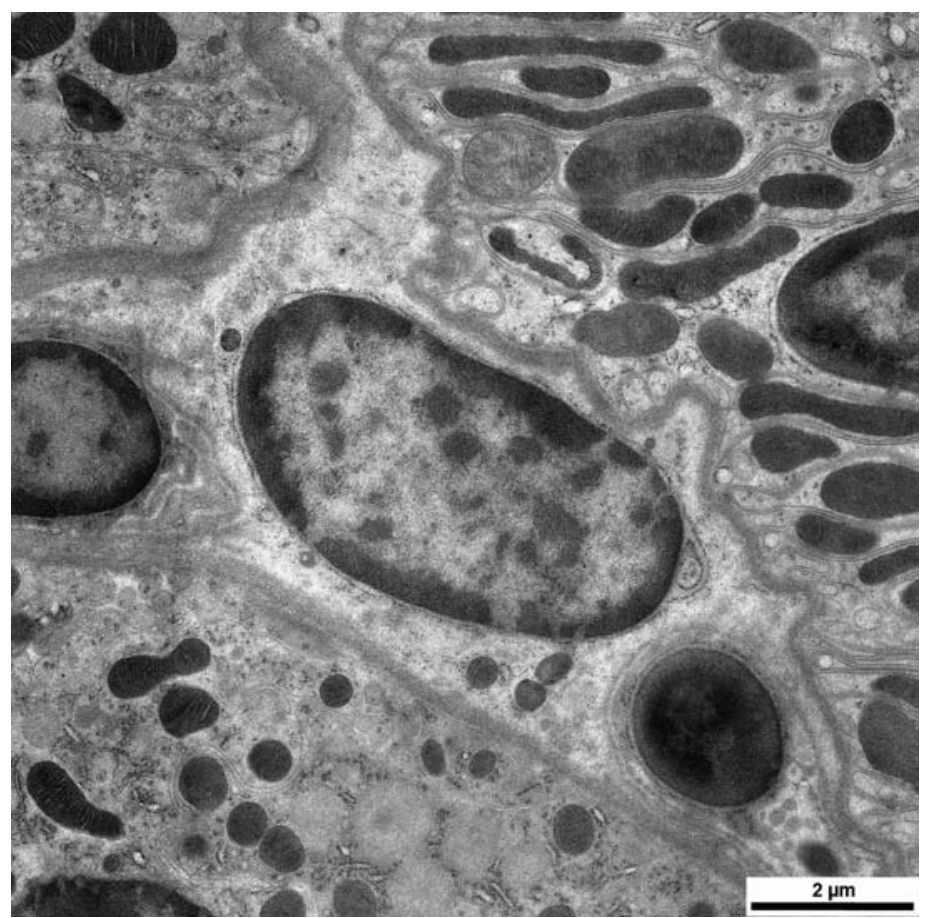

Figure 8: Kidney biopsy: Thin section of mouse kidney biopsy. Highpressure frozen in its native state (no chemical pretreatment) with a Bal-tec HPM 010 high-pressure freezer, freeze-substituted in acetone with $2 \% \mathrm{OsO}_{4}$, embedded in Epon/Araldite, thin sectioned and stained with uranyl acetate $2 \%$ and Reynolds lead citrate. Courtesy (AK) Electron Microscopy Center Zurich (EMEZ), ETH Zurich, Switzerland. without needing to resort to the use of any cryoprotectants. Cells, tissues, cultures, solutions, and many plant and animal species have been successfully frozen by this technique. Samples as large as $2 \mathrm{~mm}$ in diameter and up to $0.2 \mathrm{~mm}$ in thickness yield well-frozen 'vitreous' regions with high information density. Ice crystals of very small size are often observed, but they are minimized in the best-frozen samples. HP frozen materials can be post-processed by all the standard routines of freeze substitution and ultramicrotomy. However, freeze fracture replica production from HP frozen samples has proved to be challenging. The effects of high pressure per se on biological samples are generally minimal, although experimental controls are recommended. The versatility of this technique, and its ability to accommodate a wide range of sample types of various sizes and depths, accounts for the widespread use and popularity of the HP method. (Figure 6, 7, 8)

The original design of Moor, et al, (www.RMCProducts.com) remains the 'gold standard' among HP freezing machines, by virtue of the overwhelming number of quality publications it has yielded from expert users, as well as by the large worldwide distribution of these instruments that have already been installed. Several HP instruments are commercially available (HPM010 from RMC-Abra; others from Leica, Wohwend). Over the years, several different metal-block "slam"-freezing units of varying performance have also been produced (MedVac, Escaig, LifeCell, Polaron, Gentleman Jim, etc); however, today only Heuser's units are readily available. Propane Jet models were once manufactured and sold by RMC and Balzers/Bal-Tec. A variety of plunge freezing units, home made and commercial (Gatan, EM Science, FEI-Pella, Leica) can be found in labs worldwide

In the end, the primary goal of biological EM has always been to provide high-resolution information about cell and molecular structure, at specific times and under specific physiological conditions. The inextricably interrelated steps of specimen preparation that must all be combined to realize this goal of what we like to call "native state analysis" clearly include today some sort of cryo immobilization or cryo fixation.

Loss of information content due to improper freezing can never be restored. Moreover, even the best-frozen sample can be destroyed if improperly stored, or if improperly handled during all the subsequent preparation steps such as freeze substitution, freeze fracture and cryoimaging. The quest for excellence at each and every one of these distinct operations thus remains the surest path to accurate interpretation and successful, highly informative EM results (https://listhost.uchicago.edu/ mailman/listinfo/cryopreservation).

\section{References:}

1. Gilkey, J.C., and Staehlin, L.A. (1986). Advances in ultrarapid freezing for the preservation of cell ultrastructure. J. Electron Microsc. Tech. 3: 177-210.

2. Rothenberg, H., E. Krishnan, G. Ratter. 1997. Cryo- and Micro- Techniques: Future tools for Electron Microscopy. Journal of Computer-Assisted Microscopy. 9: 69-72.

3. Dubochet, J., et al. 1988. Cryoelectron microscopy of vitrified specimens. Quarterly Review of Biophysics. 21. 129-228.

4. Plattner, H., W.M. Fischer, W.W. Schmitt, L. Bachmann. 1972. Freeze Etching of cells without cryoprotectants. J. Cell Biology. 53: 116-126.

5. Heuser, J.E., T.S. Reese, D.M.D. Landis. 1976. Preservation of synaptic structure by rapid freezing. Cold Spring Harbor Symp. Quant. Biol. 40: 17-24.

6. Muller, M., N. Meister, H. Moor. 1980. Freezing in a Propane Jet and its application in Freeze-Fracturing. Mikroskopie. 36. 129-140.

7. Moor, $\mathrm{H}$. Theory and practice of high pressure freezing. 1987. Cryotechniques in Biological Electron Microscopy (ed. R.A. Steinbrecht \& K. Zierold). 175 191. Springer-Verlag, Berlin.

8. Kanno, H., Speedy, R.J., and Angell, C.A. Supercooling of water to -92 degrees C under pressure, Science, 189, 880, 1975. 